\title{
Impact of Endothelial 18-kDa Translocator Protein on the Quantification of ${ }^{18}$ F-DPA-714
}

\author{
Catriona Wimberley ${ }^{1}$, Sonia Lavisse ${ }^{2}$, Vincent Brulon ${ }^{1}$, Marie-Anne Peyronneau ${ }^{1}$, Claire Leroy ${ }^{1}$, Benedetta Bodini ${ }^{3}$, \\ Philippe Remy ${ }^{2,4}$, Bruno Stankoff ${ }^{3}$, Irène Buvat ${ }^{1}$, and Michel Bottlaender ${ }^{1,5}$ \\ ${ }^{I} I M I V$, Inserm, CEA, Université Paris-Sud, Université Paris Saclay, Orsay, France; ${ }^{2}$ MIRCen, CEA and CNRS-UMR9199, Université \\ Paris-Sud, Fontenay-aux-Roses, France; ${ }^{3}$ Institut du Cerveau et de la Moelle épinière, Inserm, U 1127, F-75013, Paris, France, and \\ Sorbonne University, UPMC Université Paris 06, UMR S 1127, Paris, France; ${ }^{4}$ Neurology Department, Centre Expert Parkinson, and \\ NeurATRIS, CHU Henri Mondor, AP-HP and Université Paris-Est Créteil, France; and ${ }^{5}$ Neurospin, CEA, Gif-sur-Yvette, France
}

\begin{abstract}
${ }^{18} \mathrm{~F}-\mathrm{DPA}-714$ is a second-generation tracer for PET imaging of the 18-kDa translocator protein (TSPO), a marker of neuroinflammation. Analysis and interpretation of TSPO PET are challenging, especially because of the basal expression of TSPO. The aim of this study was to evaluate a compartmental model that accounts for the effect of endothelial TSPO binding on the quantification of ${ }^{18} \mathrm{~F}$-DPA-714 PET scans from a cohort of healthy subjects. Methods: Fifteen healthy subjects (9 high-affinity binders and 6 mixed-affinity binders) underwent ${ }^{18} \mathrm{~F}-\mathrm{DPA}-714$ PET scans with arterial blood sampling and metabolite analysis. The kinetic parameters were quantified using a 2-tissue compartmental model (2TC) as well as a 2TC with an extra, irreversible, compartment for endothelial binding (2TC-1K). These regional parameters and messenger RNA (mRNA) expression specific to endothelial cells were correlated with regional TSPO mRNA expression. Results: The $2 \mathrm{TC}-1 \mathrm{~K}$ model was more appropriate than the $2 \mathrm{TC}$ for $81 \%$ of fits. The total volume of distribution was significantly reduced by $21 \% \pm$ $12 \%$ across all regions with the $2 \mathrm{TC}-1 \mathrm{~K}$, compared with the $2 \mathrm{TC}$. The endothelial binding parameter $K_{b}$ varied highly across brain regions. $K_{b}$ strongly and significantly correlated with all 3 probes extracted for TSPO mRNA expression ( $r=0.80, r=0.79$, and $r=0.90)$, but no correlation was seen with the other binding parameters from the 2TC$1 \mathrm{~K}$. For the 2TC, there was a lower but significant correlation between the volume of distribution and one of the TSPO mRNA probes $(r=$ 0.65). A strong, significant correlation was seen between mRNA for TSPO and genes specific to endothelial cells. Conclusion: Accounting for endothelial TSPO in the kinetic model improved the fit of PET data. The high correlation between $K_{b}$ and TSPO mRNA suggests that the $2 \mathrm{TC}-1 \mathrm{~K}$ model reveals more biologic information about the regional density of TSPO than the 2TC. The correlation between TSPO and endothelial cell mRNA supports the relationship between the regional variation of $K_{b}$ and endothelial TSPO. These results can improve the estimation of binding parameter estimates from ${ }^{18} \mathrm{~F}-\mathrm{DPA}-714 \mathrm{PET}$, especially in diseases that induce vascular change.
\end{abstract}

Key Words: PET; neuroinflammation; TSPO; ${ }^{18} \mathrm{~F}-\mathrm{DPA}-714$; quantification

J Nucl Med 2018; 59:307-314

DOI: 10.2967/jnumed.117.195396

Received May 2, 2017; revision accepted Jul. 7, 2017.

For correspondence or reprints contact: Catriona Wimberley, IMIV, Service Hospitalier Frédéric Joliot, 4, place du General Leclerc, 91401 Orsay Cedex, France.

E-mail: catriona.wimberley@cea.fr

Published online Aug. 3, 2017.

COPYRIGHT (C 2018 by the Society of Nuclear Medicine and Molecular Imaging.
$\mathbf{T}$ he $18-\mathrm{kDa}$ translocator protein (TSPO) is a five-transmembrane domain protein predominantly located on the outer membrane of mitochondria $(1,2)$. This protein is present in peripheral tissues and in the central nervous system. TSPO has a role in the immune and inflammatory response, being upregulated by microglia in an activated state after a brain injury (3). This characteristic makes TSPO a biomarker of interest for neuroinflammation in the study of neurodegenerative and neurologic diseases such as Alzheimer disease, Parkinson disease, multiple sclerosis, and epilepsy (4).

Several PET radioligands that target TSPO have been developed, beginning with ${ }^{11} \mathrm{C}-\mathrm{PK} 11195$. A second generation of TSPO ligands for PET was developed (5), among which the most used are ${ }^{11} \mathrm{C}-\mathrm{PBR}-28,{ }^{18} \mathrm{~F}-\mathrm{PBR}-111$, and ${ }^{18} \mathrm{~F}-\mathrm{DPA}-714$ ( $N, N$-diethyl-2-[4(2-fluoroethoxy)phenyl]-5,7-dimethylpyrazolo[1,5- $a$ ]pyrimidine3-acetamide).

The initial evaluation of ${ }^{18} \mathrm{~F}$-DPA-714 in humans used the Logan plot to calculate kinetic parameters, reporting that the tracer could provide a sensitive measure of neuroinflammatory changes (6). Since then, 2 main studies have characterized the kinetic parameter estimates of ${ }^{18} \mathrm{~F}$-DPA-714 in healthy subjects using modeling with 2 tissue compartments (2TC, Fig. 1A) and metabolite-corrected arterial input functions $(7,8)$.

The analysis and interpretation of PET scans is challenging for all second-generation TSPO tracers for several reasons (9). One relates to the genetic polymorphism yielding 3 different levels of affinity (10)- high-, mixed-, and low-affinity binders-which ideally should be taken into consideration when analyzing PET scans.

Another challenge is the ubiquitous and nonnegligible TSPO expression present within healthy brain tissue. Increasing evidence has shown that this basal TSPO is not solely in the quiescent microglia and astrocytes but also in the endothelial cells of the blood-brain barrier. For example, Zhang et al. performed a messenger RNA (mRNA) expression analysis of the different cell types of the mouse cerebral cortex (11), and a searchable mRNA database is available online (https://web.stanford.edu/group/barres_ lab/brain_rnaseq.html) showing that endothelial cells have the same levels of TSPO mRNA as microglia. Furthermore, several immunohistochemistry studies in the mouse, nonhuman primate, and human have shown colocalization of endothelial cell and TSPO markers (12-18). 

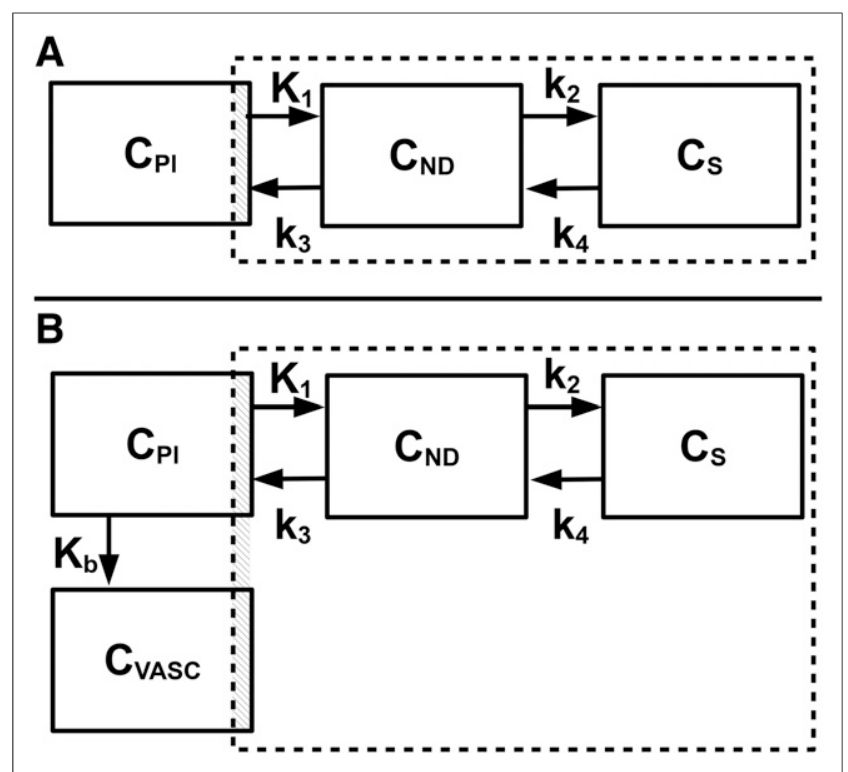

FIGURE 1. 2TC (A) and 2TC-1K (B) models (proposed by Rizzo et al. (19)). Each compartment represents pool of radioligand concentration: $\mathrm{C}_{\mathrm{PL}}$ (concentration within plasma), $\mathrm{C}_{\mathrm{ND}}$ (nondisplaceable concentration), $\mathrm{C}_{\mathrm{S}}$ (concentration specifically bound within brain tissue), and $\mathrm{C}_{\mathrm{VASC}}$ (concentration within vascular compartment). Dotted line indicates concentrations captured by PET scan, and hatched area indicates vascular fraction.

Given the evidence in the literature for the nonnegligible presence of endothelial TSPO, a kinetic model that includes a separate component to account for this binding should be tested for TSPO tracers. So far, 2 kinetic models have been developed accounting for the TSPO within the neurovasculature. The first, developed for ${ }^{11} \mathrm{C}-\mathrm{PK}-11195$ (14), modified the simplified reference tissue model to include a vascular signal, which was estimated directly from the image. The second, for ${ }^{11} \mathrm{C}-\mathrm{PBR}-28$, used the classic 2TC model and added an extra, irreversible, compartment within the vascular volume to separate the vascular TSPO binding from the tissue TSPO binding, as shown in Figure 1B (2TC-1K model) (19). The authors demonstrated that for ${ }^{11} \mathrm{C}$ PBR-28, it was necessary to include a compartment for the vascular trapping of the ligand to avoid a significant bias in the estimated binding parameters.

The purpose of our study was to evaluate the $2 \mathrm{TC}-1 \mathrm{~K}$ model when applied to ${ }^{18} \mathrm{~F}-\mathrm{DPA}-714$ scans in healthy subjects $(8)$ and to compare the results with those from the 2TC model to observe the impact of incorporating a compartment to account for endothelial TSPO. Furthermore, we investigated whether the estimated binding parameters agreed with regional TSPO within brain tissue as predicted by mRNA data extracted from the Allen Human Brain (AHB) atlas (20).

\section{MATERIALS AND METHODS}

\section{Study Design and Imaging Protocol}

Fifteen healthy volunteers ( 8 women and 7 men; mean age \pm SD, $50 \pm 16$ y) were included from 2 clinical protocols (NCT02305264 and NCT02319382). The study protocols were approved by a Medical Bioethics Committee of the Île de France Region and in accordance with French legislation and the 1975 Declaration of Helsinki (revised in 1983), and written informed consent was obtained from all participants. All individuals were genotyped for the s6971 polymorphism within the TSPO gene, yielding 9 high-affinity binders and 6 mixed-affinity binders. Each participant underwent T1-weighted MRI (for region-of-interest definition) and ${ }^{18} \mathrm{~F}-\mathrm{DPA}-714$ PET scans (ECAT High-Resolution Research Tomograph; Siemens Medical Solutions). An ${ }^{18} \mathrm{~F}-\mathrm{DPA}-714$ bolus was injected intravenously (192 \pm $21 \mathrm{MBq}$ ), and the dynamic list-mode PET acquisition lasted $90 \mathrm{~min}$. During the acquisition, arterial blood sampling and metabolism measurements were performed to generate an arterial input function. The demographic details, genetic analysis, metabolite analysis, ${ }^{18}$ F-DPA-714 synthesis, and imaging protocols were described by Lavisse et al. (8).

\section{Reconstruction and Image Processing}

Images were reconstructed using the expectation-maximization maximum-likelihood algorithm with 40 iterations, point-spread function modeling, and postsmoothing $(21,22)$. The 33 time frames for the dynamic scan were $1 \times 20,10 \times 10,3 \times 20,2 \times 60,5 \times 120,9 \times 300$, and $3 \times 600 \mathrm{~s}$, and the images had an isotropic voxel size of $1.2 \mathrm{~mm}^{3}$. After reconstruction, each frame was corrected for motion using PMOD software (version 3.6; PMOD Technologies Ltd.). All T1weighted MR images were segmented using FreeSurfer, version 5.3 (https://surfer.nmr.mgh.harvard.edu/), producing a neuroanatomic atlas that was registered with the PET scans for region-of-interest definition and time-activity curve extraction. Data from the left and right regions were averaged.

The ECAT High-Resolution Research Tomograph, dedicated to brain imaging, has an intrinsic isotropic spatial resolution of about $2.5 \mathrm{~mm}$ and a sensitivity of about $5.5 \%-6.5 \%$ (23); hence, dynamic scans are strongly affected by noise. For noise reduction, a principalcomponent analysis was applied (Pixies software, http://www.apteryx. $\mathrm{fr} /$ ) in which the largest signals in the image described by the first 3 principal components were conserved and the rest were discarded as noise $(24,25)$. Supplemental Figure 1 (supplemental materials are available at http://jnm.snmjournals.org) shows the curves taken from a large region (high-count data with little noise) and a small region before and after noise reduction, showing the impact of the process. A simulation study (results not shown) has also indicated that this noise reduction does not induce a bias in the dynamic scan in regions of high or low noise. The time-activity curve extraction and kinetic modeling were done for the original images and the images with noise reduction.

\section{Kinetic Modeling}

Our previous study ( 8 ) showed that the $2 \mathrm{TC}$ model was more appropriate than a $1 \mathrm{TC}$ model; in the current study, therefore, only the 2TC model (Fig. 1A) was compared with the 2TC-1K model (Fig. 1B). A nonlinear least-squares model fitting was applied using the PMOD software implementation. The microparameters estimated were the rate constants between the compartments: $K_{1}$ $\left(\mathrm{mL} / \mathrm{cm}^{3} / \mathrm{min}\right), k_{2}, k_{3}$, and $k_{4}\left(\mathrm{~min}^{-1}\right)$. The $K_{b}\left(\mathrm{~min}^{-1}\right)$ parameter, which describes the rate of binding to the TSPO in the endothelium, and the vascular fraction (vB in Supplemental Tables 2-7) were also estimated. The macroparameters estimated were total volume of distribution $\left(\mathrm{V}_{\mathrm{T}}\right)\left(\mathrm{mL} / \mathrm{cm}^{3}\right), \mathrm{V}_{\mathrm{ND}}$ (nondisplaceable volume of distribution $\left.\left[\mathrm{mL} / \mathrm{cm}^{3}\right]\right)$, and $\mathrm{BP}_{\mathrm{ND}}$ as $k_{3} / k_{4}$ (unitless).

\section{Correlation of PET Parameter Estimates with mRNA Expression}

The mRNA expression data can be extracted from the freely available AHB atlas and used for comparison with protein or receptor concentration maps acquired with PET (26). The comparison is made under the assumption that mRNA expression can predict in vivo protein distribution. A correlation has already been 


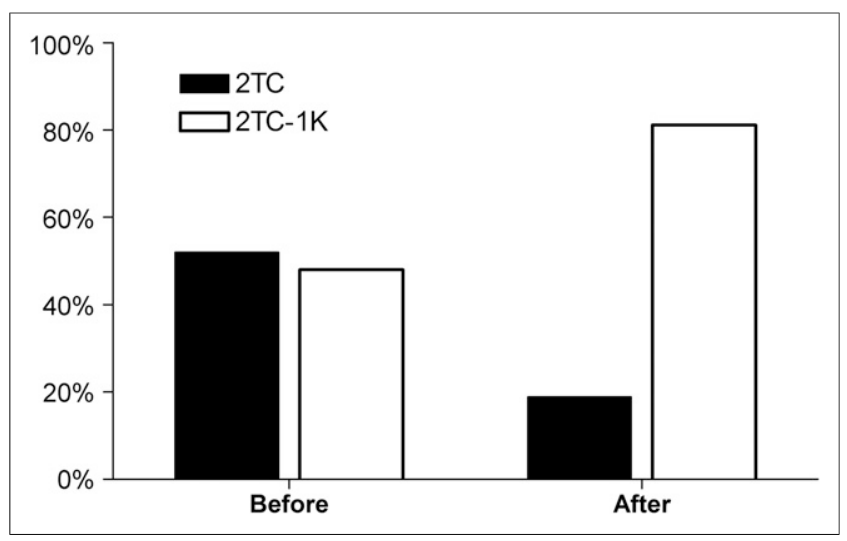

FIGURE 2. Percentage of $2 \mathrm{TC}$ and $2 \mathrm{TC}-1 \mathrm{~K}$ time-activity curves preferred by AIC for each of the 2 models before and after noise reduction.

demonstrated between TSPO mRNA and protein expression (27). In the AHB atlas, the expressed mRNA was measured using a large range of genetic probes, including 3 different probes for the TSPO gene (Supplemental Table 1) and many probes for genes specifically expressed within endothelial cells. In this study, the kinetic parameter estimates from the PET scans were compared with mRNA TSPO expression. The mRNA data were extracted at the regional level and correlated with the regional macroparameters in both models for the same regions when available.

The connection between TSPO and endothelial markers was investigated by correlating the TSPO mRNA with mRNA data extracted for 4 genes known to be specific within endothelial cells (vascular endothelial zinc finger 1, FMS-related tyrosine kinase 1, forkhead box Q1, and platelet/endothelial cell adhesion molecule 1;
Supplemental Table 1) (28). The correlations were done at a region level and averaged over the different probes for each endothelial gene.

\section{Statistical Analysis}

The compartmental models were compared using the model-fitting criterion (PMOD software). The model selection comparison used the Akaike information criterion (AIC) corrected for a small sample size and the Bayesian information and model selection criteria (MicroMath). Kinetic parameter estimates are presented as mean $\pm \mathrm{SD}$, and subjects were grouped by genetic affinity. The SE of each parameter estimate, showing the precision of the estimate, is expressed as a percentage of the parameter itself and defined as the coefficient of variation. The regional parameter estimates between high- and mixedaffinity binders were compared using a 1-way ANOVA. Parameter differences between the 2 models were compared using the MannWhitney $U$ test. Pearson correlation coefficients $(r)$ were calculated to evaluate the correlation between estimated parameters and mRNA expression. Significance was set at a $P$ value of less than 0.05 .

\section{RESULTS}

\section{Model Assessment and Noise Reduction}

The AIC values from the original images showed a similar percentage of time-activity curves that fit better for both models (Fig. 2). It was not possible to distinguish which of the 2 models provided the best fit (average AIC, $-3 \pm 14$ for the $2 \mathrm{TC}$ and $-7 \pm 16$ for the $2 \mathrm{TC}-1 \mathrm{~K}$ over all regions). After noise reduction, the $2 \mathrm{TC}-1 \mathrm{~K}$ yielded a better fit than the $2 \mathrm{TC}$ for $81 \%$ of all time-activity curves (Fig. 2) according to the AIC. The mean AIC values improved $(-38 \pm 24$ for the $2 \mathrm{TC}$ and $-50 \pm 29$ for the $2 \mathrm{TC}-1 \mathrm{~K}$, all regions), and the difference in the AIC between the 2 models was significant for 12 of the 15 subjects.

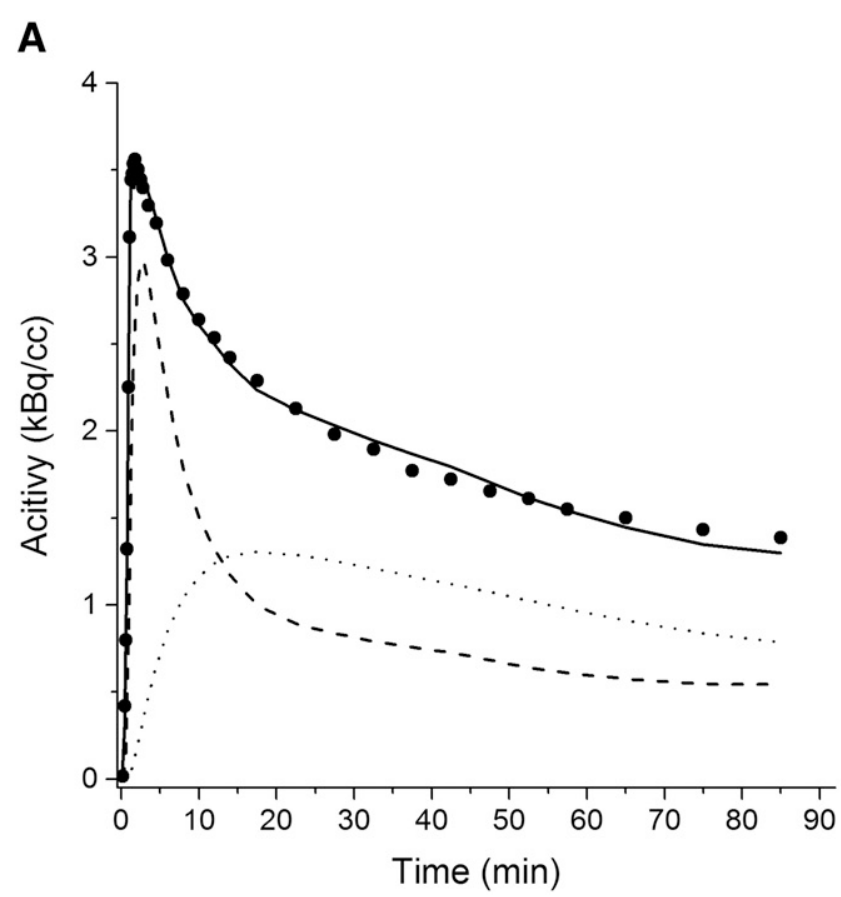

B

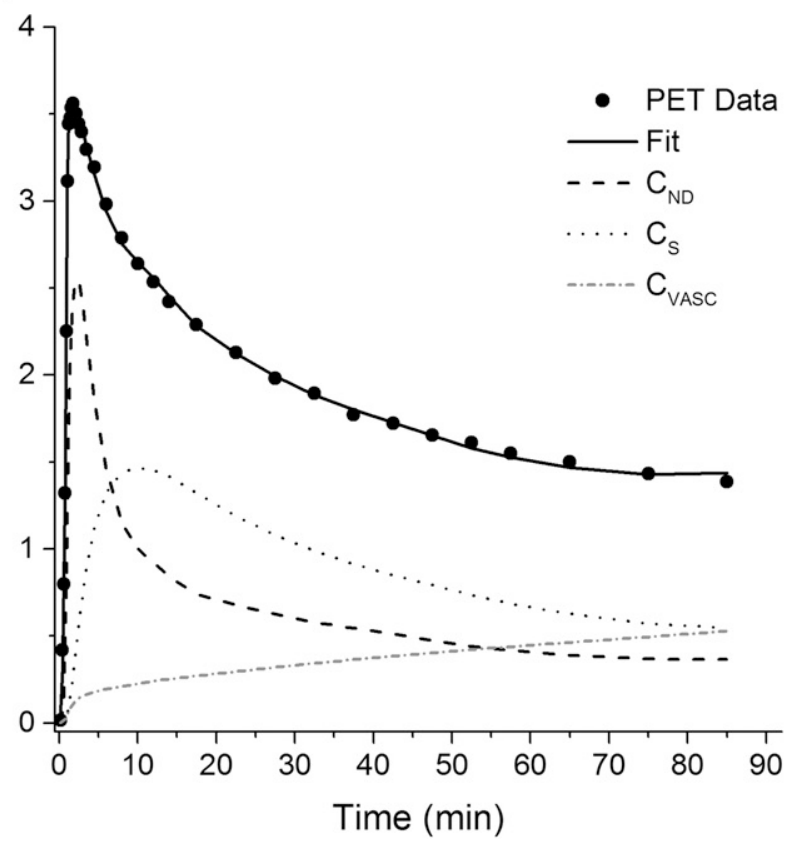

FIGURE 3. Model fits for 1 representative time-activity curve with $2 \mathrm{TC}(\mathrm{A})$ and $2 \mathrm{TC}-1 \mathrm{~K}(\mathrm{~B})$ models. $\mathrm{C}_{\mathrm{ND}}=$ nondisplaceable concentration, $\mathrm{C}_{\mathrm{S}}=$ concentration specifically bound within brain tissue, $\mathrm{C}_{\mathrm{VASC}}=$ concentration within vascular compartment. 
A thorough analysis was undertaken to assess whether noise removal induced a bias in the time-activity curves and model fitting. Details on changes in the AIC in a large and small region are described in Supplemental Figure 1. In large regions with little to no noise, the time-activity curve values, shape, and AIC stayed the same, leading to the inference that no bias was induced. All subsequent results were thus obtained from the images after noise reduction.

The model fits of the $2 \mathrm{TC}$ and $2 \mathrm{TC}-1 \mathrm{~K}$ are shown in Figure 3 for a representative whole-brain time-activity curve. The fit using the $2 \mathrm{TC}-1 \mathrm{~K}$ model followed the measured data more closely, especially at the middle and end of the time-activity curve, as was reflected in the improved AIC (Fig. 2).
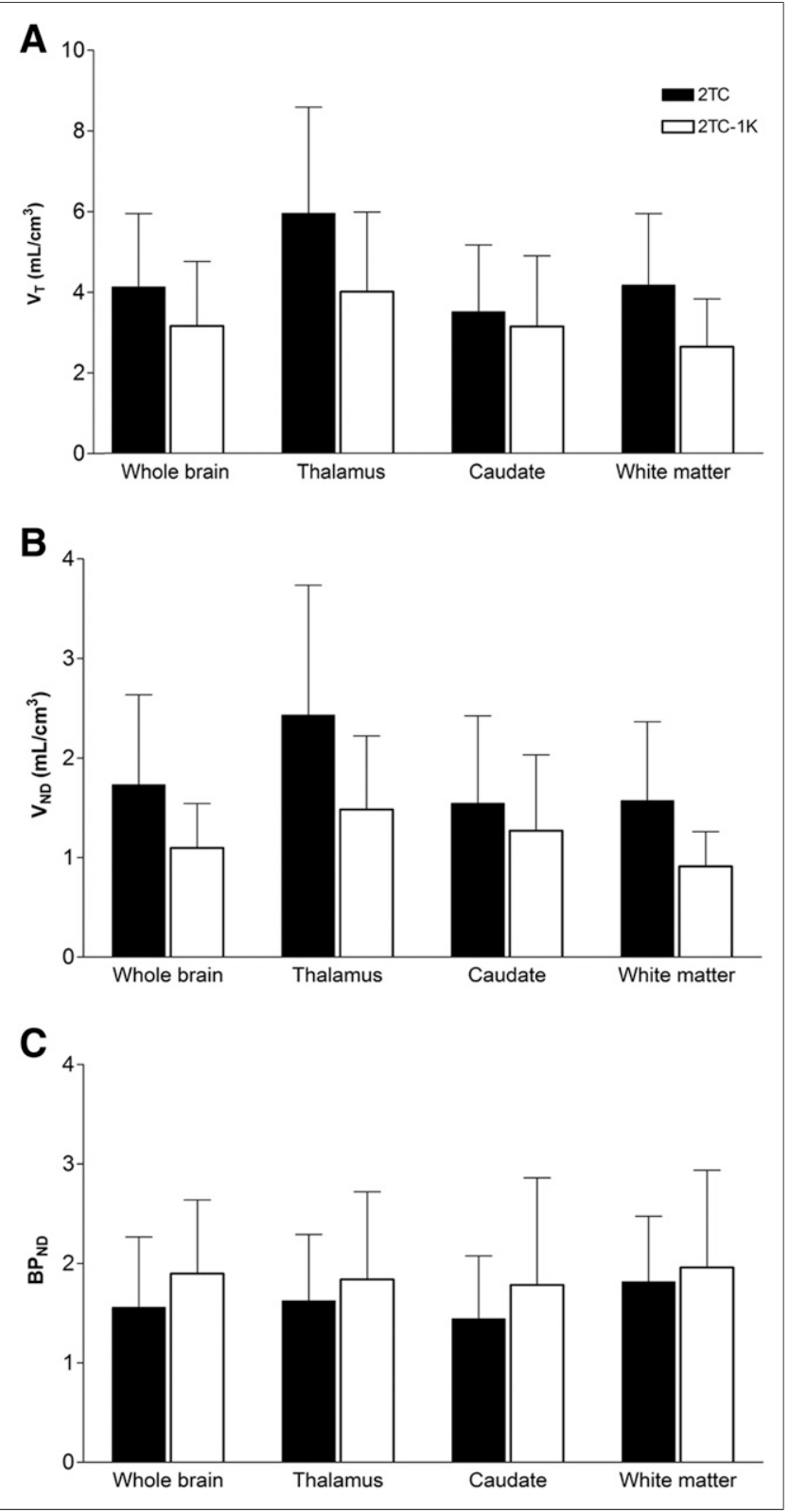

FIGURE 4. Mean parameter estimates for $2 \mathrm{TC}$ and $2 \mathrm{TC}-1 \mathrm{~K}$ models in some brain regions of high-affinity-binders. Parameters shown are $V_{T}$ $(\mathrm{A}), \mathrm{V}_{\mathrm{ND}}(\mathrm{B})$, and $B \mathrm{P}_{\mathrm{ND}}(\mathrm{C})$.
The other model-fitting criteria that we tested were more favorable for the 2TC-1K than for the 2TC: the Bayesian information criterion identified a better fit for the $2 \mathrm{TC}-1 \mathrm{~K}$ than for the $2 \mathrm{TC}$ for $84 \%$ of the curves, and the model selection criterion identified a better fit for the $2 \mathrm{TC}-1 \mathrm{~K}$ than for the $2 \mathrm{TC}$ for $91 \%$ of the curves.

\section{Kinetic Parameters}

The parameter estimates for both models are shown in Figure 4 for representative regions. The average estimates for vascular fraction, $\mathrm{V}_{\mathrm{T}}, \mathrm{V}_{\mathrm{ND}}, \mathrm{BP}_{\mathrm{ND}}$, and $K_{b}$ are shown in Supplemental Tables 27. The 1-way ANOVA showed a significant effect of the affinity of the subjects (high- or mixed-affinity binders) for the macroparameters estimated $\left(\mathrm{V}_{\mathrm{T}}, \mathrm{V}_{\mathrm{ND}}, \mathrm{BP}_{\mathrm{ND}}\right.$, and $\left.K_{b}\right)$ for both models $(F$ statistic between 12 and 53, $P<0.05$ ).

The $2 \mathrm{TC}-1 \mathrm{~K}$ model produced $\mathrm{V}_{\mathrm{T}}$ and $\mathrm{V}_{\mathrm{ND}}$ values significantly lower than those from the $2 \mathrm{TC}$ model in all regions (average reduction, $21 \% \pm 12 \%$ ). The $\mathrm{BP}_{\mathrm{ND}}$ showed higher estimates using the $2 \mathrm{TC}-1 \mathrm{~K}$ for all regions; however, differences between the models were not significant in any region. When the $2 \mathrm{TC}$ model was used, the thalamus showed the highest $\mathrm{V}_{\mathrm{T}}$ and the caudate showed the lowest. When the $2 \mathrm{TC}-1 \mathrm{~K}$ model was used, the thalamus still had the highest $\mathrm{V}_{\mathrm{T}}$ value, but the lowest was in the white matter. The $K_{b}$ was quite variable across the brain regions analyzed, as seen in Figure 5, and the average regional $K_{b}$ correlated strongly between high- and mixed-affinity binders $(r=0.97$, $P<0.05)$.

The parameters of the 2TC model had an average regional coefficient of variation of $2 \pm 2$ and $12 \pm 5$ for $\mathrm{V}_{\mathrm{T}}$ and $\mathrm{BP}_{\mathrm{ND}}$, respectively. For the $2 \mathrm{TC}-1 \mathrm{~K}$ model, the average regional coefficient of variation was $4 \pm 3$ and $18 \pm 10$ for $\mathrm{V}_{\mathrm{T}}$ and $\mathrm{BP}_{\mathrm{ND}}$, respectively. The average regional coefficient of variation for $K_{b}$ was $26 \pm 23$.

\section{Correlations with mRNA Data}

The regional correlations between the TSPO mRNA expression and the estimated PET macroparameters (Fig. 6) were significant for the $\mathrm{V}_{\mathrm{T}}$ from the $2 \mathrm{TC}$ for only TSPO probe 3 . $K_{b}$ from the $2 \mathrm{TC}$ $1 \mathrm{~K}$ strongly and significantly correlated with all 3 TSPO gene probes. The correlations between TSPO and endothelial mRNA expression, as shown in Figure 7, were strong and significant.

\section{DISCUSSION}

Recently, there has been increasing evidence that TSPO is present in cells other than microglia and astrocytes, such as endothelial cells, which can affect the quantification and interpretation of PET studies $(9,13,16-19)$. We investigated the impact of adding an extra compartment into the 2TC model to account for ${ }^{18} \mathrm{~F}$-DPA-714 binding to endothelial TSPO in the blood-brain barrier. We showed that the model with this extra compartment fit the PET data much better according to modelfitting criteria; however, this improvement was not clear until the noise in the dynamic scans was reduced. Further, the parameter describing tracer binding to endothelial TSPO $\left(K_{b}\right)$ correlated strongly with TSPO mRNA data extracted from the online AHB atlas, indicating that the $2 \mathrm{TC}-1 \mathrm{~K}$ model reveals information about the regional density of TSPO.

A large proportion of noise could be removed, improving the identification of the models in small regions with high noise. The shape of the kinetic time-activity curves remained unchanged in the large regions (with little to no noise) after noise reduction, nor 


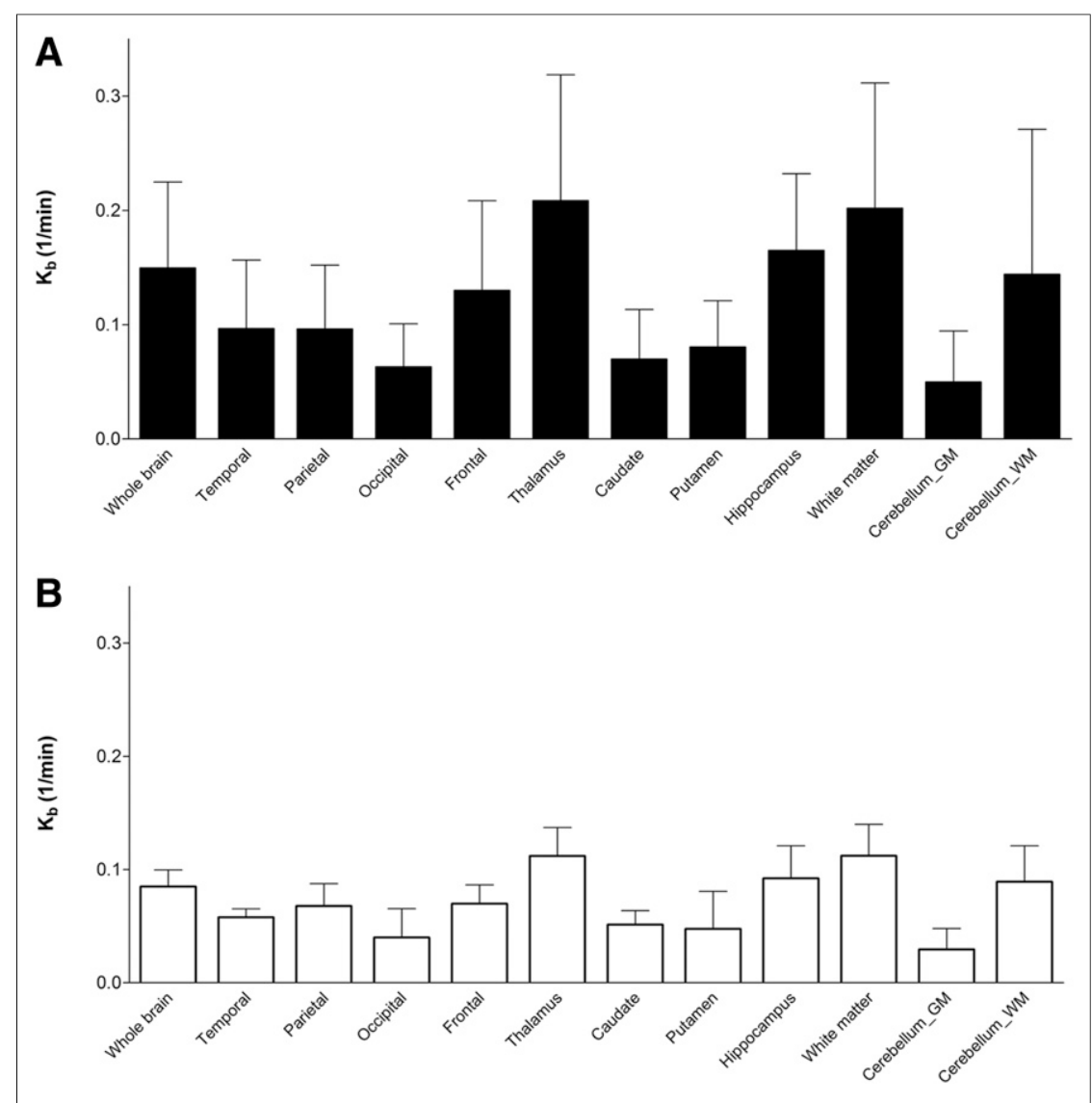

FIGURE 5. Mean $K_{b}$ estimates for $2 \mathrm{TC}-1 \mathrm{~K}$ model for each brain region for high-affinity $(\mathrm{A})$ and mixed-affinity $(B)$ binders. $\mathrm{GM}=$ gray matter; $\mathrm{WM}=$ white matter. obtained using the $2 \mathrm{TC}-1 \mathrm{~K}$ may be more relevant to the microglial or astrocytic TSPO expression involved with neuroinflammatory processes than those estimated by the 2TC. For accurate interpretation of the data, it is especially important to separate these sources of TSPO when there is a pathology involving the endothelium, such as vascular system degradation as demonstrated in Alzheimer disease (14) or vascular enhancement as demonstrated in some gliomas or endothelial inflammation (17).

A significant correlation was found between 1 of the 3 TSPO mRNA probes and the $\mathrm{V}_{\mathrm{T}}$ value estimated using the 2TC. Strong and significant correlations were found between the estimated $K_{b}$ of the $2 \mathrm{TC}-1 \mathrm{~K}$ and all $3 \mathrm{TSPO}$ probes, but no correlation was found between the 2TC$1 \mathrm{~K} \mathrm{~V}_{\mathrm{T}}$ or $\mathrm{BP}_{\mathrm{ND}}$ and these probes. These strong correlations demonstrate that the 2TC-1K model reveals more information about the regional variation in TSPO than the 2TC and that the $K_{b}$ describes the main TSPO variation between brain regions in healthy subjects. The correlation between the regional $K_{b}$ estimates and the TSPO mRNA is consistent with the fact that little to no activated microglia are present in healthy brain tissue. The main TSPO signal may therefore come from other sources, such as the endothelial cells.

To further investigate the relationship between $K_{b}$ and endothelial TSPO conwas the AIC changed, suggesting that this process did not introduce bias.

Adding the extra compartment related to the vascular component clearly improved the fitting as demonstrated by the AIC, showing that the $2 \mathrm{TC}-1 \mathrm{~K}$ is a more appropriate model for $81 \%$ of the time-activity curves. The model used was one originally proposed by Rizzo et al. (19) with irreversible binding within the endothelial compartment. It is possible that the component is actually slowly reversible, but the vascular washout parameter of the endothelial TSPO would be difficult to identify from an experiment with a single-injection tracer dose. The true interaction of the tracer with endothelial TSPO, compared with tissue TSPO, is unknown; therefore, an additional parameter to account for the vascular washout was not introduced and an irreversible compartment was used to estimate the shape of the kinetic of the endothelial binding.

The impact of using the $2 \mathrm{TC}-1 \mathrm{~K}$ model can be seen as a reduction in the average $V_{T}$ and $V_{N D}$ values in all regions for all subjects. Interestingly, the regional reduction of both $\mathrm{V}_{\mathrm{T}}$ and $\mathrm{V}_{\mathrm{ND}}$ correlated strongly with the added $K_{b}$ parameter $(r=0.96, P<$ $0.05) . \mathrm{BP}_{\mathrm{ND}}$ increased slightly with the $2 \mathrm{TC}-1 \mathrm{~K}$, but the increase was not significant and the regional trend was similar to the regional $\mathrm{BP}_{\mathrm{ND}}$ estimated by the $2 \mathrm{TC}$ and not strongly variable between brain regions. Because the $\mathrm{V}_{\mathrm{T}}$ from the $2 \mathrm{TC}$ model consists partly of endothelial binding, use of the $2 \mathrm{TC}-1 \mathrm{~K}$ removes this constitutive binding, suggesting that the binding parameters centration, we extracted the mRNA data related to TSPO, as well as the mRNA data for 4 genes specifically expressed within endothelial cells. The high regional correlation that could be seen between these 2 targets (Fig. 7) supports the hypothesis that the variation in TSPO expression in healthy subjects is related to endothelial TSPO. There were several genes specific to endothelial cells in the AHB atlas (28), but some of them showed no significant correlation with TSPO mRNA. This finding could be because the presence of mRNA expression does not provide a direct estimation of the protein present in the tissue but can support the presence of the protein if there is a correlation (26).

The evidence for TSPO within the endothelium is further supported by a recent blocking study of ${ }^{11} \mathrm{C}-\mathrm{PBR}-28$ PET showing that the $2 \mathrm{TC}-1 \mathrm{~K}$ model was more appropriate than the $2 \mathrm{TC}$ model. In this study, immunohistochemistry showed that TSPO was present in all vessels and accounted for $30 \%$ of the vascular network and that this variation in TSPO was dependent on tissue type (29).

The regional correlations between parameter estimates and TSPO mRNA expression are in line with previous data (19) showing that $K_{b}$ correlated well with TSPO mRNA expression, although less strongly than with ${ }^{18} \mathrm{~F}-\mathrm{DPA}-714$. Unlike what was seen for ${ }^{18} \mathrm{~F}$-DPA-714, a correlation was present between the TSPO mRNA and the $\mathrm{V}_{\mathrm{T}}$ of the $2 \mathrm{TC}-1 \mathrm{~K}$ for ${ }^{11} \mathrm{C}-\mathrm{PBR}-28$. This correlation could be due to the different affinity of the tracer, 


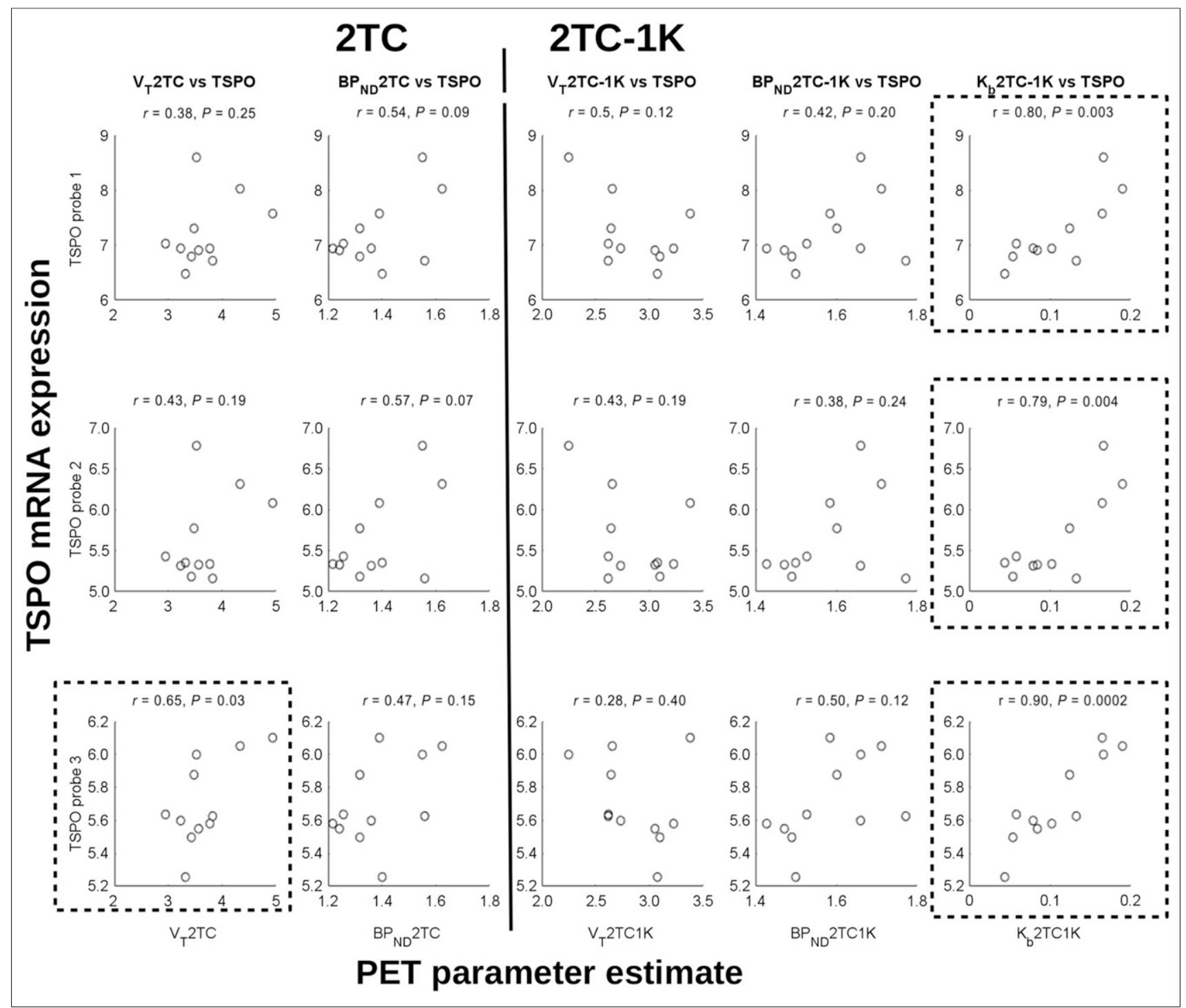

FIGURE 6. Correlations of kinetic parameter estimates (all subjects) from ${ }^{18} \mathrm{~F}-\mathrm{DPA}-714$ PET scans with TSPO mRNA expression from AHB atlas. (Left) $\mathrm{V}_{\mathrm{T}}$ and $\mathrm{BP}_{\mathrm{ND}}$ from 2TC. (Right) $\mathrm{V}_{\mathrm{T}}, \mathrm{BP}_{\mathrm{ND}}$, and $K_{b}$ from $2 \mathrm{TC}-1 \mathrm{~K}$. Plots surrounded by box show significant correlations.

making it challenging to separate the different signals with a single tracer dose.

To the best of our knowledge, there have been only 2 other studies using TSPO tracers that tested the 2TC-1K model $(30,31)$. Fan et al. (30) showed that ${ }^{18} \mathrm{~F}-\mathrm{GE} 180$, in healthy subjects, fit better with the $2 \mathrm{TC}$ than with the $2 \mathrm{TC}-1 \mathrm{~K}$. However, the behavior and kinetics of this tracer are quite different from those of ${ }^{18} \mathrm{~F}$ DPA-714, having comparatively low $\mathrm{V}_{\mathrm{T}}$ values, and therefore a different model choice and estimates can be expected. Bloomfield et al. (31), using ${ }^{11} \mathrm{C}-\mathrm{PBR}-28$ in a cohort of schizophrenic patients, reported that the $2 \mathrm{TC}-1 \mathrm{~K}$ fit better for $99.4 \%$ of time-activity curves based on the AIC.

On the basis of our findings, ${ }^{18} \mathrm{~F}-\mathrm{DPA}-714$ PET kinetic parameters derived without accounting for endothelial binding should be interpreted with caution, especially in neurologic diseases that include a component of vascular degeneration, angiogenesis, or inflammation $(14,17)$. Quantification using reference region methods and not accounting for the regionally variable $K_{b}$ could under- or overestimate $\mathrm{BP}_{\mathrm{ND}}$. Finally, separation of tracer binding to endothelial TSPO from tissue TSPO binding may increase the sensitivity with which small changes in TSPO expression due to neuroinflammatory processes are detected.

\section{CONCLUSION}

Kinetic modeling of ${ }^{18} \mathrm{~F}-\mathrm{DPA}-714$ in the healthy human revealed that an extra compartment is required to account for the nonnegligible binding to the TSPO present in the endothelial cells of the blood-brain barrier. The best model could be identified only after noise was reduced, highlighting the need to have low-noise data for accurate kinetic modeling. The model choice was supported by evidence from the literature showing TSPO within the endothelium and the strong correlation of the endothelial binding parameter, $K_{b}$, with TSPO mRNA. The regional variation in $K_{b}$ explained by endothelial TSPO was consistent with correlations between TSPO mRNA 


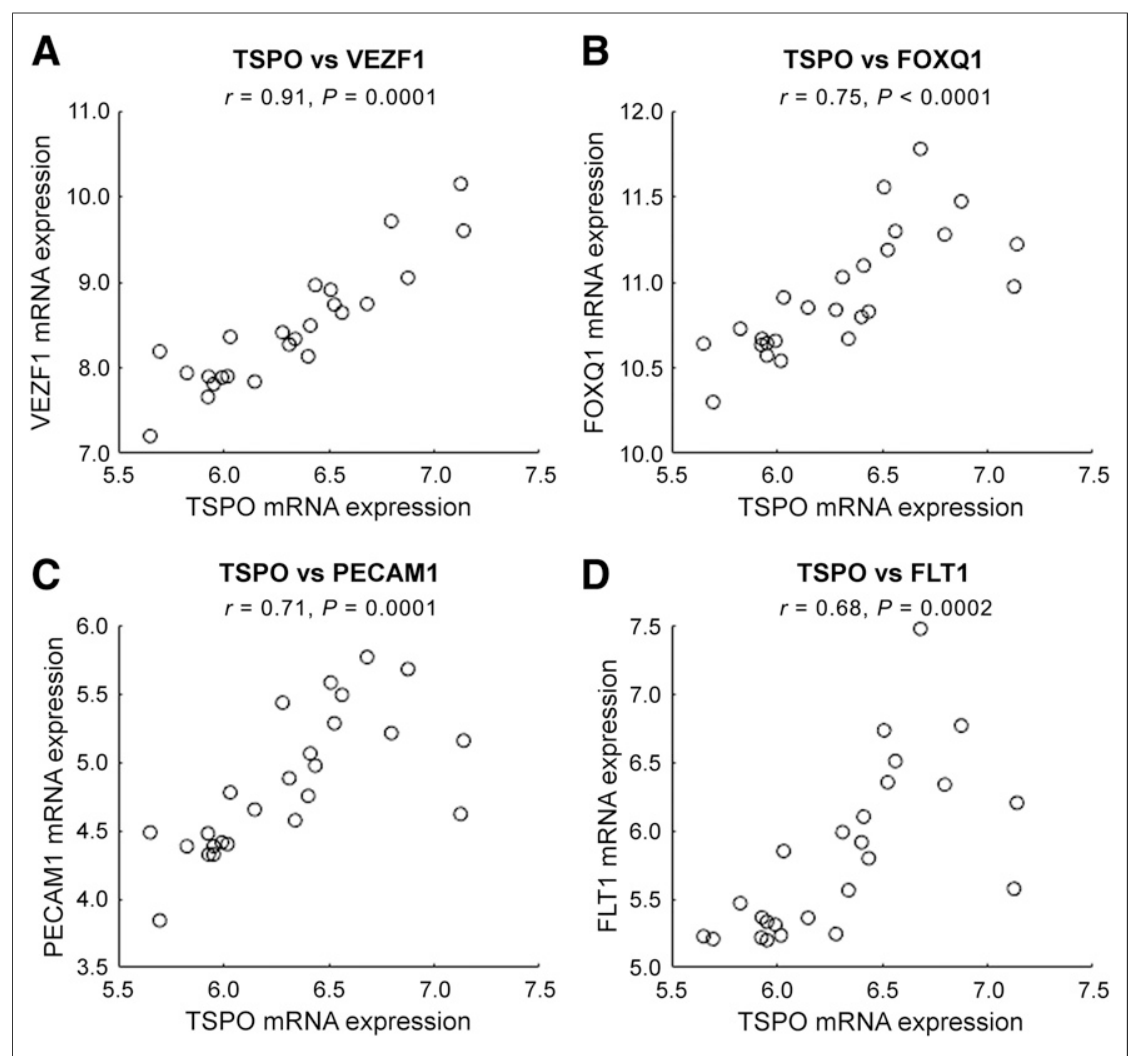

FIGURE 7. Regional correlations over 25 different regions from AHB atlas between average TSPO mRNA expression ( $x$-axis) and mRNA expression for 4 different genes specific to endothelial cells ( $y$-axis): vascular endothelial zinc finger 1 (VEZF1) (A), forkhead box Q1 (FOXQ1) (B), platelet/endothelial cell adhesion molecule 1 (PECAM1) (C), and FMS-related tyrosine kinase 1 (FLT1) (D).

and the mRNA specific to endothelial cells. These results should affect the interpretation of binding parameter estimates from ${ }^{18} \mathrm{~F}$-DPA-714 PET in diseases that include a component of vascular change.

\section{DISCLOSURE}

The work was performed at the SHFJ imaging facility supported by France Life Imaging under grant agreement ANR-11-INSB-006. This work was supported by France Parkinson (NCT02319382), ANR-08-MNPS-016-02 and ANR-10-IAIHU-06 (NCT02305264), and "Investissement d'Avenir-ANR-11-INBS-0011"-NeurATRIS, CEA. Catriona Wimberley was supported by Eurotalents postdoctoral grant 600382 . This study was partially funded by the EU 7th framework programme (FP7/2007-2013) under grant agreement 278850 (INMiND). No other potential conflict of interest relevant to this article was reported.

\section{ACKNOWLEDGMENTS}

We are grateful to the SHFJ staff: the nurses and the Molecular Probes Team of IMIV, Claude Comtat, Simon Stute, and Philippe Gervais. We thank Lena Guillot, Isabelle Rebeix, Sylvie Forlani, and Luce Dauphinot for the genetic analysis, and we thank Gaia Rizzo, Mattia Veronese, and Carole Escartin for fruitful discussions.

\section{REFERENCES}

1. Anholt RR, Pedersen PL, De Souza EB, Snyder SH. The peripheral-type benzodiazepine receptor: localization to the mitochondrial outer membrane. $J$ Biol Chem. 1986;261:576-583.

2. Anholt RR, De Souza EB, Oster-Granite ML, Snyder SH. Peripheral-type benzodiazepine receptors: autoradiographic localization in whole-body sections of neonatal rats. J Pharmacol Exp Ther. 1985; 233:517-526.

3. Venneti S, Lopresti BJ, Wiley CA. The peripheral benzodiazepine receptor (translocator protein $18 \mathrm{kDa}$ ) in microglia: from pathology to imaging. Prog Neurobiol. 2006;80:308-322.

4. Rupprecht R, Papadopoulos V, Rammes G, et al. Translocator protein $(18 \mathrm{kDa})(\mathrm{TSPO})$ as a therapeutic target for neurological and psychiatric disorders. Nat Rev Drug Discov. 2010;9:971988.

5. Chauveau F, Boutin H, Van Camp N, Dollé F, Tavitian B. Nuclear imaging of neuroinflammation: a comprehensive review of $\left[{ }^{11} \mathrm{C}\right] \mathrm{PK} 11195$ challengers. Eur J Nucl Med Mol Imaging. 2008; 35:2304-2319.

6. Arlicot $\mathrm{N}$, Vercouillie $\mathrm{J}$, Ribeiro $\mathrm{MJ}$, et al. Initial evaluation in healthy humans of ${ }^{18} \mathrm{~F}-\mathrm{DPA}-714$, a potential PET biomarker for neuroinflammation. $\mathrm{Nucl}$ Med Biol. 2012;39:570-578.

7. Golla SS, Boellaard R, Oikonen V, et al. Quantification of $\left[{ }^{18} \mathrm{~F}\right] \mathrm{DPA}-714$ binding in the human brain: initial studies in healthy controls and Alzheimer's disease patients. J Cereb Blood Flow Metab. 2015;35:766-772.

8. Lavisse S, Garcia-Lorenzo D, Peyronneau MA, et al. Optimized quantification of translocator protein radioligand ${ }^{18} \mathrm{~F}$-DPA-714 uptake in the brain of genotyped healthy volunteers. J Nucl Med. 2015;56: $1048-1054$

9. Turkheimer FE, Rizzo G, Bloomfield PS, et al. The methodology of TSPO imaging with positron emission tomography. Biochem Soc Trans. 2015;43: 586-592.

10. Owen DR, Howell OW, Tang SP, et al. Two binding sites for $\left[{ }^{3} \mathrm{H}\right] \mathrm{PBR} 28$ in human brain: implications for TSPO PET imaging of neuroinflammation. $J$ Cereb Blood Flow Metab. 2010;30:1608-1618.

11. Zhang Y, Chen K, Sloan SA, et al. An RNA-sequencing transcriptome and splicing database of glia, neurons, and vascular cells of the cerebral cortex. $J$ Neurosci. 2014;34:11929-11947.

12. Mirzaei N, Tang SP, Ashworth S, et al. In vivo imaging of microglial activation by positron emission tomography with $\left[{ }^{11} \mathrm{C}\right] \mathrm{PBR} 28$ in the 5XFAD model of Alzheimer's disease. Glia. 2016;64:993-1006.

13. Roncaroli F, Su Z, Herholz K, Gerhard A, Turkheimer FE. TSPO expression in brain tumours: is TSPO a target for brain tumour imaging? Clin Transl Imaging. 2016;4:145-156.

14. Tomasi G, Edison P, Bertoldo A, et al. Novel reference region model reveals increased microglial and reduced vascular binding of ${ }^{11} \mathrm{C}-(\mathrm{R})$ PK11195 in patients with Alzheimer's disease. J Nucl Med. 2008;49:12491256.

15. Turkheimer FE, Edison $\mathrm{P}$, Pavese N, et al. Reference and target region modeling of $\left[{ }^{11} \mathrm{C}\right]-(R)-\mathrm{PK} 11195$ brain studies. J Nucl Med. 2007;48:158167.

16. Cosenza-Nashat M, Zhao ML, Suh HS, et al. Expression of the translocator protein of $18 \mathrm{kDa}$ by microglia, macrophages and astrocytes based on immunohistochemical localization in abnormal human brain. Neuropathol Appl Neurobiol. 2009;35:306-328

17. Notter T, Coughlin JM, Gschwind T, et al. Translational evaluation of translocator protein as a marker of neuroinflammation in schizophrenia. Mol Psychiatry. January 17, 2017 [Epub ahead of print].

18. Kanno I, Seki C, Takuwa H, et al. Positron emission tomography of cerebral angiogenesis and TSPO expression in a mouse model of chronic hypoxia. $J$ Cereb Blood Flow Metab. January 1, 2017 [Epub ahead of print].

19. Rizzo G, Veronese M, Tonietto M, Zanotti-Fregonara P, Turkheimer FE, Bertoldo A. Kinetic modeling without accounting for the vascular component 
impairs the quantification of $\left[{ }^{11} \mathrm{C}\right] \mathrm{PBR} 28$ brain PET data. J Cereb Blood Flow Metab. 2014;34:1060-1069.

20. Hawrylycz MJ, Lein ES, Guillozet-Bongaarts AL, et al. An anatomically comprehensive atlas of the adult human brain transcriptome. Nature. 2012;489:391399.

21. Sibomana M, Keller S, Stute S. Benefits of 3D scatter correction for the HRRT: a large axial FOV PET scanner. NSS/MIC: IEEE Nucl Sci Symp Med Imaging Conf Rec. 2013;2954-2957.

22. Stute S, Comtat C. Practical considerations for image-based PSF and blobs reconstruction in PET. Phys Med Biol. 2013;58:3849-3870.

23. Sossi V, de Jong HWAM, Barker WC, et al. The second generation HRRT: a multi-centre scanner performance investigation. IEEE Nucl Sci Symp Conf Rec. 2005;4:2195-2199.

24. Tsartsalis S, Moulin-Sallanon M, Dumas N, et al. Quantification of GABAA receptors in the rat brain with $\left.{ }^{123} \mathrm{I}\right]$ iomazenil SPECT from factor analysisdenoised images. Nucl Med Biol. 2014;41:186-195.

25. Millet P, Moulin-Sallanon M, Tournier BB, et al. Quantification of dopamine $D_{2 / 3}$ receptors in rat brain using factor analysis corrected $\left[{ }^{18} \mathrm{~F}\right]$ fallypride images. Neuroimage. 2012;62:1455-1468.

26. Rizzo G, Veronese M, Heckemann RA, et al. The predictive power of brain mRNA mappings for in vivo protein density: a positron emission tomography correlation study. J Cereb Blood Flow Metab. 2014;34: 827-835.

27. Canat X, Carayon P, Bouaboula M, et al. Distribution profile and properties of peripheral-type benzodiazepine receptors on human hemopoietic cells. Life Sci. 1993;52:107-118.

28. Daneman R, Zhou L, Agalliu D, et al. The mouse blood-brain barrier transcriptome: a new resource for understanding the development and function of brain endothelial cells. PLoS One. 2010;5:e13741.

29. Veronese M, Reis Marques T, Bloomfield PS, et al. Kinetic modelling of $\left[{ }^{11} \mathrm{C}\right] P B R 28$ for $18 \mathrm{kDa}$ translocator protein PET data: a validation study of vascular modelling in the brain using XBD173 and tissue analysis. J Cereb Blood Flow Metab. January 1, 2017 [Epub ahead of print].

30. Fan Z, Calsolaro V, Atkinson RA, et al. Flutriciclamide ( $\left.{ }^{18} \mathrm{~F}-\mathrm{GE} 180\right)$ PET: firstin-human PET study of novel third-generation in vivo marker of human translocator protein. J Nucl Med. 2016;57:1753-1759.

31. Bloomfield PS, Selvaraj S, Veronese M, et al. Microglial activity in people at ultra high risk of psychosis and in schizophrenia: an $\left[{ }^{11} \mathrm{C}\right]$ PBR28 PET brain imaging study. Am J Psychiatry. 2016;173:44-52. 